\title{
Author Correction: Ion channels as lipid sensors: from structures to mechanisms
}

Mackenzie J. Thompson (D) and John E. Baenziger (D)

Correction to: Nature Chemical Biology https://doi.org/10.1038/s41589-020-00693-3, published online 16 November 2020

In the version of this Review Article originally published, there was an error in the description of a cited reference in the "Lipid-gated $\mathrm{K}^{+}$channels" section of the text. In the sentence "Although crystal structures suggest that both $\mathrm{PIP}_{2}$ and G-protein binding (the latter mimicked by the R201A mutation) are required to open the inner helix gate (PDB: 3SYQ; $3.5 \AA$ resolution) ${ }^{29}$, recent cryo-EM structures solved in lipid nanodiscs suggest that $\mathrm{PIP}_{2}$ binding alone translates the cytoplasmic domain up toward the TM pore domain, as in Kir2.2 $\left(\right.$ ref. ${ }^{30}$ )," 'lipid nanodiscs' should be changed to 'detergent' to read "... recent cryo-EM structures solved in detergent...". The error has been corrected.

Published online: 17 December 2020

https://doi.org/10.1038/s41589-020-00722-1

(c) The Author(s), under exclusive licence to Springer Nature America, Inc. 2020 